\title{
Characterisation of the new EpCAM-specific antibody HO-3: implications for trifunctional antibody immunotherapy of cancer
}

\author{
P Ruf ${ }^{*, 1}$, O Gires ${ }^{2}$, M Jäger', K Fellinger ${ }^{2,5}, \mathrm{~J} \mathrm{Atz}^{3}$ and H Lindhofer ${ }^{1,4}$ \\ 'Department of Antibody Development, TRION Research GmbH, Martinsried, Germany; ${ }^{2}$ Clinical Cooperation Group Molecular Oncology, GSF-Research \\ Centre for Health, and Environment and Department of Otorhinolaryngology, Ludwig-Maximilians-University, Munich, Germany; ${ }^{3}$ Department of \\ Preclinical Research and Development, Fresenius Biotech $\mathrm{GmbH}$, Gräfelfing, Germany; ${ }^{4} \mathrm{CEO}, \mathrm{TRION}$ Pharma GmbH, Munich, Germany
}

Epithelial cell adhesion molecule EpCAM is a transmembrane glycoprotein that is frequently overexpressed in a variety of carcinomas. This pan-carcinoma antigen has served as the target for a plethora of immunotherapies. Innovative therapeutic approaches include the use of trifunctional antibodies (trAbs) that recruit and activate different types of immune effector cells at the tumour site. The trAb catumaxomab has dual specificity for EpCAM and CD3. In patients with malignant ascites, catumaxomab significantly increased the paracentesis-free interval, corroborating the high efficacy of this therapeutic antibody. Here, we characterised the monoclonal antibody ( $\mathrm{mAb}$ ) HO-3, that is, the EpCAM-binding arm of catumaxomab. Peptide mapping indicated that $\mathrm{HO}-3$ recognises a discontinuous epitope, having three binding sites in the extracellular region of EpCAM. Studies with glycosylation-deficient mutants showed that $\mathrm{mAb} \mathrm{HO}-3$ recognised EpCAM independently of its glycosylation status. High-affinity binding was not only detected for $\mathrm{mAb} \mathrm{HO}-3$, but also for the monovalent EpCAM-binding arm of catumaxomab with an excellent $K_{\mathrm{D}}$ of $5.6 \times 10^{-10} \mathrm{M}$. Furthermore, trAb catumaxomab was at least a 1000-fold more effective in eliciting the eradication of tumour cells by effector peripheral blood mononuclear cells compared with mAb HO-3. These findings suggest the great therapeutic potential of trAbs and clearly speak in favour of EpCAM-directed cancer immunotherapies.

British Journal of Cancer (2007) 97, 315-321. doi:10.1038/sj.bjc.660388 I www.bjcancer.com

Published online 10 July 2007

(c) 2007 Cancer Research UK

Keywords: EpCAM (CD326); immunotherapy; trifunctional antibodies

EpCAM (CD326) is a type I transmembrane glycoprotein that acts as a $\mathrm{Ca}^{2+}$ independent homophilic cell adhesion molecule (Litvinov et al, 1994). The $39-42 \mathrm{kDa}$ protein is composed of a large extracellular domain with two EGF-like repeats, a single transmembrane region, and a short cytoplasmic tail of 26 amino acids (Balzar et al, 1999). The high frequency of its overexpression in a multitude of human carcinomas qualifies EpCAM as a target of interest for immunotherapy. High-level EpCAM expression was demonstrated in major malignancies such as colon, stomach, and prostate cancers and in adenocarcinoma of the lung (Went et al, 2006). Besides being a target antigen, EpCAM is a prognostic marker in breast, ovarian, and gallbladder cancers where its overexpression correlates with poor prognosis (Gastl et al, 2000; Spizzo et al, 2002, 2006; Varga et al, 2004). The therapeutic potential for the inhibition of EpCAM was illustrated by a knockdown phenotype of EpCAM in carcinoma cells. Treatment of head neck, and breast cancer cells with EpCAM-specific antisense or siRNAs resulted in the reduction or even abrogation of cell proliferation, migration, and invasive capacity (Munz et al, 2004; Osta et al, 2004).

\footnotetext{
*Correspondence: Dr P Ruf, TRION Research GmbH, Am Klopferspitz 19,82 I52 Martinsried, Germany; E-mail: peter.ruf@trionresearch.de ${ }^{5}$ Current address: LMU Biozentrum, Martinsried, Germany.

Received I5 March 2007; revised 6 June 2007; accepted 19 June 2007; published online 10 July 2007
}

Moreover, EpCAM supports active escape from immune surveillance. Ectopic expression of EpCAM in dendritic cells blocks their ability to present MHC class II-restricted antigens (Gutzmer et al, 2004). All these features have encouraged the development of various EpCAM-directed anti-cancer therapies. Trifunctional antibodies (trAbs) with a dual specificity for EpCAM expressed on tumour cells and $\mathrm{CD} 3$ expressed on $\mathrm{T}$ cells represent an innovative immunotherapeutic approach to fight cancer. TrAbs induce a tricell complex of tumour cells, $\mathrm{T}$ cells, and accessory immune cells due to their unique Fc-composition of mouse IgG2a and rat IgG2b. Thereby, crosstalk between different types of redirected immune effector cells is initialised, that results in efficient killing of the tumour cells (Zeidler et al, 1999, 2000; Riesenberg et al, 2001). Encouraging first clinical results confirm those observed in in vitro anti-tumour potency studies. Positive indication of clinical benefit in the treatment of metastatic breast cancer came from a phase I dose escalation trial using a HER-2-specific trAb (Kiewe et al, 2006). Moreover, the feasibility of combining trAbs with high-dose chemotherapy for metastatic breast cancer treatment was shown in a pilot study (Stemmler et al, 2005). Finally, trAbs were safely applied and demonstrated convincing efficacy in patients suffering from malignant ascites (Heiss et al, 2005).

Here, we report on the characterisation of the new monoclonal antibody (mAb) HO-3 that constitutes the EpCAM-binding arm of the corresponding trAb catumaxomab (removab ${ }^{\mathbb{R}}$ ). A detailed analysis of the binding features and anti-tumour efficacy of HO-3 
in comparison with catumaxomab is presented. Furthermore, we discuss the impact of the binding kinetics with respect to immunotherapy of EpCAM-positive tumours by conventional monoclonal $v s$ trifunctional antibodies.

\section{MATERIALS AND METHODS}

\section{Generation of HO-3 hybridoma}

$\mathrm{Balb} / \mathrm{c}$ mice were immunised by intraperitoneal injection of the EpCAM-positive human colon carcinoma cell line HCT-8 (ATCC No. CCL-224). One week after a booster immunisation, the mice were killed and spleen cell preparations were fused with the mouse myeloma cell line P3X63Ag8.653 (ATCC No. CRL 1580). Supernatants of single growing clones were screened for competitive binding with anti-EpCAM mAb C215 to HCT-8 cells by flow cytometry. The isolated hybridoma clone HO-3 was further stabilised by several rounds of subcloning. It produces mouse antibodies of the subtype IgG2a.

\section{Antibodies and EpCAM antigen}

The EpCAM-specific antibodies C215 (Bjork et al, 1993), VU-1D9 (Balzar et al, 1999), HO-3 and the anti-mouse Thy-1.2. isotype control MmT1 (Kremmer et al, 1989) were all produced by hybridoma technology and protein G (GE Healthcare, Uppsala, Sweden) affinity purification at TRION Research. Catumaxomab $\left(\right.$ removab ${ }^{\circledR}$ ), the trAb variant of HO-3 with a dual specificity for EpCAM and CD3 and the trifunctional control antibody Bi20 (antiCD20 $\times$ anti-CD3) were manufactured using the quadroma technology (Lindhofer et al, 1995) at TRION Pharma (Munich, Germany) under GMP conditions.

The extracellular domain (ECD) of the human EpCAM protein was heterologously expressed in the yeast strain pichia pastoris at GenYouIn Biotech (Reutlingen, Germany). C-terminal addition of a His $_{6}$ tag allowed metal-affinity purification of the protein via $\mathrm{Ni}$ NTA agarose (Qiagen, Hilden, Germany).

\section{Establishing EpCAM glycosylation mutants}

Asparagine residues within the NGT/S N-glycosylation consensus sequence at amino-acid positions 74, 111, and 198 were changed to alanines by PCR-based site directed mutagenesis. A triple knockout mutant was generated by sequential single mutations using the primers shown in Table 1. PCR amplification products were subcloned into the PCR cloning vector pDRIVE (Qiagen) before transfer into the eukaryotic expression vector pcDNA3.1-hygro ${ }^{+}$ (Invitrogen, Heidelberg, Germany) for transient transfection of HEK293 cells. Empty vector was used as a negative control for transfection and subsequent detection by flow cytometry. The glycosylation status of the proteins was assessed with the glycostain kit (Molecular Probes, Göttingen, Germany) according to the manufacturer's instructions.

\section{Construction of an EGF-like domain I EpCAM deletion mutant}

Deletion of the EGF-like domain I (amino acids 27-59) was performed by overlapping PCR mutagenesis with the primers listed in Table 1. PCR fragments were subcloned into the pDRIVE vector and subsequently transferred into pcDNA3.1-hygro ${ }^{+}$using the Nhe1 and Xbal restriction enzyme sites. Transiently transfected HEK293 cells, including a positive control plasmid expressing wild-type EpCAM, were then analysed by flow cytometry and immunoblot. Similar transfection efficiency for each sample was assessed with the GFP expression plasmid pEGFP-C1 (BD Clontech, Heidelberg, Germany).

\section{Fluorescence-activated cell sorting analysis}

Competitive binding to native EpCAM and binding to the above mentioned EpCAM glycosylation mutants was performed by flow cytometry using a fluorescence-activated cell sorting (FACS)Calibur device and the Cellquest pro software (Becton Dickinson, Heidelberg, Germany). Competitive binding assays were performed by preincubation of $2 \times 10^{5}$ HCT- 8 target cells with EpCAM specific mAbs C215 or VU-1D9 at the indicated amounts for $10 \mathrm{~min}$. After a washing step with FACS buffer (PBS supplemented with $1 \%$ FCS and $0.1 \%$ sodium azide) cells were incubated with $2.5 \mu \mathrm{g} \mathrm{ml}^{-1}$ FITC-labelled $\mathrm{HO}-3 \mathrm{mAb}$ for $30 \mathrm{~min}$, washed, and analysed by comparing the mean fluorescence intensity (MFI) relative to a mouse IgG2a, kappa isotype control (BD Pharmingen).

Analysis of HO-3 or $\mathrm{C} 215$ binding to native EpCAM mutants was done using HEK293 cells stably or transiently transfected with the

Table I Primers used for PCR-based site directed mutagenesis of epithelial cell adhesion molecule (EpCAM)

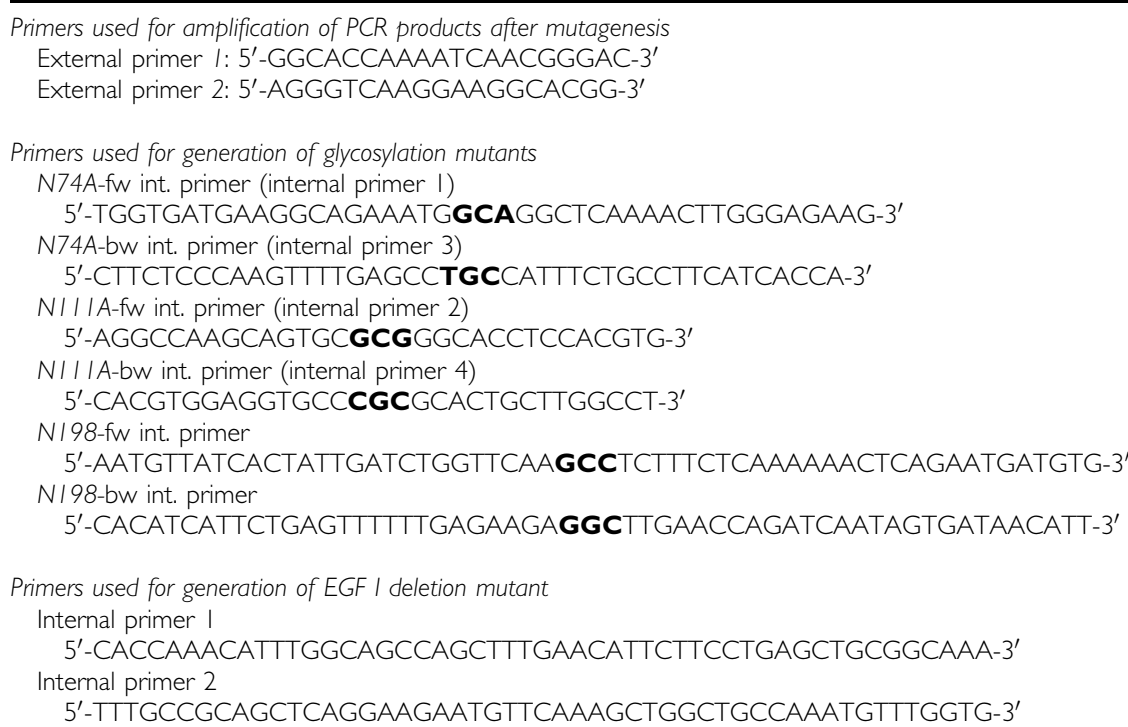


above mentioned expression vectors. Therefore, $14-20 \mu \mathrm{g} \mathrm{ml}^{-1}$ primary antibodies were each incubated with $2 \times 10^{5}$ cells for $30 \mathrm{~min}$. After washing three times, the PE or FITC-labelled rat-antimouse IgG detection antibody (Dianova, Hamburg, Germany) was added. Cells were washed again, and the MFI of the stained cells was determined.

\section{Epitope mapping}

The EpCAM epitope recognised by the HO-3 antibody was defined by screening a peptide library covering amino acids $7-268$. The 22-residue $\mathrm{N}$-acetylated peptides with a 16 amino-acid overlap were directly synthesised on a cellulose membrane by spot synthesis as described by Reineke et al (1999). After incubation with HRP-labelled HO-3 antibody, the chemiluminescence signal intensity was quantified with an imaging system as BLU (Boehringer light units). The epitope mapping studies were carried out at Jerini Peptide Technologies (Berlin, Germany).

\section{Affinity measurement}

The affinity of HO-3 and catumaxomab for the EpCAM protein was determined via surface plasmon resonance (SPR) using a Biacore 3000 device (Biacore AB, Uppsala, Sweden). The ECD of EpCAM was covalently coupled to a CM-5 sensor chip at low density (215 response units of EpCAM). Binding kinetics were performed with twofold serial dilutions of antibody at concentrations of 500-0.08 $\mathrm{nm}$ in running buffer (PBS, $\mathrm{pH} 7.4,0.005 \%(\mathrm{v} / \mathrm{v})$, polysorbate 20 - filtered and degassed) at $25^{\circ} \mathrm{C}$ and a flow rate of $25 \mu \mathrm{min}^{-1}$. The regeneration procedure consisted of three injections of $10 \mu \mathrm{l} 2.5 \mathrm{M}$ guanidinium hydrochloride followed by flushing of the sensor chip with running buffer for $5 \mathrm{~min}$. Statistical and data processing were performed with BIA evaluation software 4.0.1 and GraphPad Prism 4.02 (GraphPad Software Inc., San Diego, CA, USA). All SPR experiments were carried out at Biaffin GmbH \& Co KG (Kassel, Germany).

\section{Tetrazolium hydroxide cytotoxicity assay}

Peripheral blood mononuclear cells (PBMC) $\left(1 \times 10^{5}\right.$ cells $)$ from healthy donors were isolated by ficoll density centrifugation and subsequently mixed with $1 \times 10^{4}$ HCT- 8 tumour cells in the presence of the indicated amounts of antibodies in 96-well flat bottomed plates (final volume $200 \mu \mathrm{l}$ RPMI-1640 medium containing $10 \%$ FCS, $1 \mathrm{~mm}$ sodium pyruvate, $2 \mathrm{mM} \mathrm{L}$-glutamine, and $1 \times$ non-essential amino acids). After 3 days of co-cultivation at $37^{\circ} \mathrm{C}$ and $5 \% \mathrm{CO}_{2}$, soluble PBMC were washed twice with PBS without $\mathrm{Ca}^{2+} \mathrm{Mg}^{2+}$ (PAN Biotech, Aidenbach, Germany). Then, adherent tumour cells were stained with tetrazolium hydroxide (XTT) cell proliferation kit II (Roche Diagnostics $\mathrm{GmbH}$, Mannheim, Germany) until the $\mathrm{OD}_{650 \mathrm{~nm}-490 \mathrm{~nm}}$ of the tumour cell control samples reached a value of about 1.5. Plates were measured with a VersaMax plate reader (Molecular Devices, CA, USA) with background values with medium alone subtracted. Killing efficiency of HO-3 and catumaxomab was assessed in a total of four independent experiments with fourfold determinations of each sample. Tumour cell killing (\%) was calculated according to the formula: $\left(\mathrm{OD}_{\text {tumour }}\right.$ cells $-\mathrm{OD}_{\text {tumour }}$ cells $+\mathrm{PBMC}+$ antibody $) /$ $\left(\mathrm{OD}_{\text {tumour cells }}-\mathrm{OD}_{\mathrm{PBMC}}\right) \times 100 \%$.

\section{RESULTS}

\section{C215, but not VU-1D9, competes with HO-3 for binding to EpCAM}

To confirm the specificity of the mAb HO-3 for EpCAM, we performed binding assays in the presence of the mAbs C215 and VU-1D9. Both C215 and VU-1D9 bind with high-affinity to the

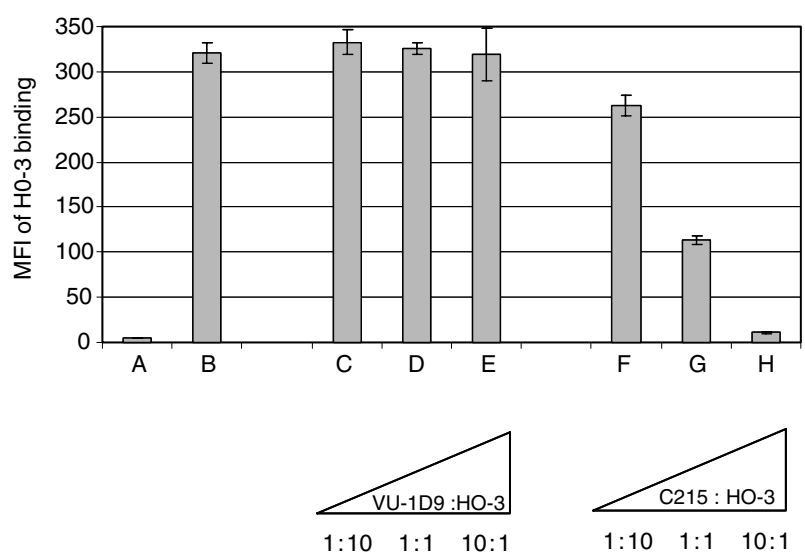

Figure I $\quad \mathrm{C} 2 \mathrm{I}$, but not $\mathrm{VU}$ - ID9, competes with $\mathrm{mAb} \mathrm{HO}-3$ for binding to wild-type EpCAM. Preincubation of HCT-8 tumour cells with $\mathrm{mAb}$ $\mathrm{C} 215$ blocked $\mathrm{HO}-3$ binding by more than $50 \%$ relative to the positive control (B) with no competitor when the antibodies were added at equal concentrations $\left(2.5 \mu \mathrm{g} \mathrm{ml}^{-1}\right)$. A I0-fold excess of mAb C215 $\left(\left.25 \mu \mathrm{g} \mathrm{ml}\right|^{-1}\right)$ resulted in almost complete binding inhibition, which was down to the level of the isotype control (A; IgG2a, kappa). Preincubation of cells with VUID9 did not significantly influence $\mathrm{HO}-3$ binding. Triangles indicate escalating amounts of competitor antibody with $\mathrm{HO}-3$ concentration kept constant at $2.5 \mu \mathrm{g} \mathrm{ml}^{-1}$. Each sample was measured three times; error bars indicate s.d. The experiment was repeated with similar results.

EGF-like domain I of EpCAM (Balzar et al, 1999). As depicted in Figure 1, C215 clearly inhibited the binding of HO-3 to EpCAMpositive HCT-8 cells in a dose-dependent manner, whereas VU-1D9 did not. A 10-fold excess of preadded mAb C215 entirely blocked HO-3 binding to EpCAM-positive cells suggesting that both antibodies recognise similar epitopes.

\section{HO-3 binds within the EGF I repeat of EpCAM}

The C215 antibody binds within the EGF-like domain I of EpCAM (Bjork et al, 1993). Given the fact that C215, but not VU-1D9, competed for the binding of HO-3 to EpCAM, we anticipated similar binding sites for $\mathrm{C} 215$ and HO-3. To test our assumption, flow cytometry assays were conducted to assess the binding activity of HO-3 to an EpCAM deletion mutant lacking the EGFlike domain I (EGFmut; amino acids 27-59). Indeed, the reactivity of HO-3 towards the deletion mutant was strongly impaired when compared to the recognition of an EpCAM wild-type control (Figure 2A). A similar loss of binding to the EGF-like domain mutant was observed for the mAb C215, which was used as a control. However, in both cases residual binding activity could be detected. A comparably strong, though not complete, loss of binding activity for mAbs HO-3 and C215 was observed when EpCAM-EGFmut was detected in its denatured form by immunoblotting (Figure 2B). These results define the binding specificity of HO-3 and C215 within EpCAM's EGF-like domain I, but point towards additional binding sites beyond this particular protein stretch.

\section{HO-3 recognises a discontinuous epitope, having three binding sites in EpCAM}

Next, the binding specificity of HO-3 was fine-mapped by screening a peptide library covering the complete ECD of EpCAM (amino acids 7-268). We analysed 22-residue peptides, with a 16-amino-acid overlap, to achieve high-resolution mapping. The synthetic peptide library included the amino acid sequence ranging from residues 31 to 52 of wild-type EpCAM that was most strongly targeted by mAb C215 (Bjork et al, 1993). Screening 

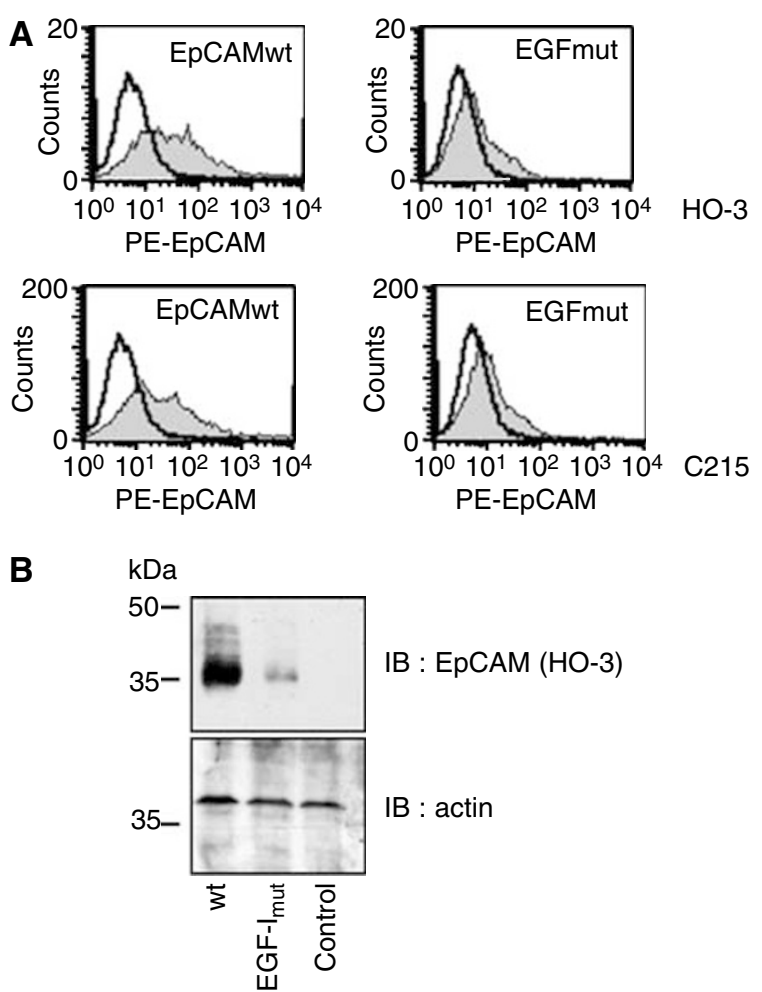

Figure 2 HO-3 binds within EGF-like domain I of EpCAM. (A) HEK293 were transiently transfected with wild-type EpCAM or the EpCAM EGF-I mutant and stained with HO-3 (grey curves; upper panels) or C2I 5 (grey curves; lower panels) in combination with a PE-conjugated secondary antibody. As a control, primary Ab was omitted (black line). (B) Transfections were performed as in (A), proteins were separated by I $0 \%$ SDS-PAGE, and EpCAM was visualised with the mAb HO-3. For a control, levels of actin were assessed on the same membrane. Shown are the representative results from three independent experiments.

the peptide library with the $\mathrm{C} 215$ control antibody confirmed the recognition of two peptides at amino acid positions $31-52$ and 103-124 (Table 2). Interestingly, mAb HO-3 recognised a conformational epitope, indicating the presence of three EpCAM binding sites. A comparison of the amino acid sequences recognised by $\mathrm{C} 215$ and $\mathrm{HO}-3$ revealed an overlap in the epitope within the EGF-like domain I. This common binding region readily explains the competitive reactivity of both investigated antibodies. However, an additional major HO-3 binding site was localised within the EGF-like domain II between amino acids 67 and 88. A third binding site situated more C-terminally (103 - 124) could also be identified. Notably, there was no cross-reaction with murine EpCAM (data not shown).

HO-3 and the corresponding trAb catumaxomab bind with high affinity to EpCAM

Binding of HO-3 to EpCAM-positive target cells, as measured by flow cytometry, pointed toward a high-affinity antibody (data not shown). Surface plasmon resonance was applied to determine the exact binding constants for HO-3. For this purpose, the recombinant ECD of native EpCAM was expressed in yeast, and the antibody-antigen interaction was investigated with Biacore technology. Both HO-3 and the corresponding trAb catumaxomab revealed high affinities, with low dissociation constants of $5.5 \times 10^{-10}$ and $5.6 \times 10^{-10} \mathrm{M}$, respectively (Table 3 ). Owing to the low EpCAM antigen density covalently linked to the sensor chip, these values mainly reflect monovalent binding. After increasing EpCAM density avidity effects of bivalent $\mathrm{HO}-3$
Table 2 Identification of EpCAM peptides recognised by $\mathrm{HO}-3$ and C215.

\begin{tabular}{llcc}
\hline $\begin{array}{l}\text { AA } \\
\text { position }\end{array}$ & Peptide sequence & $\begin{array}{c}\text { Signal intensity } \\
\text { (BLU) }\end{array}$ & mAb \\
\hline $49-70$ & TSVGAQNTVICSKLAAKCLVMK & 6829 & $\mathrm{HO}-3$ \\
$67-88$ & LVMKAEMNGSKLGRRAKPEGAL & 18813 & $\mathrm{HO}-3$ \\
$175-196$ & QLDPKFITSILYENNVITIDLV & 3543 & $\mathrm{HO}-3$ \\
$31-52$ & NYKLAVNCFVNNNRQCQCTSVG & 16703 & $\mathrm{C} 215$ \\
$103-124$ & GLFKAKQCNGTSTCWCVNTAGV & 12006 & $\mathrm{C} 215$ \\
\hline
\end{tabular}

Screening a peptide library covering the complete ECD of EpCAM showed that the listed peptides had the strongest interaction with the indicated antibody. Signals ranging from 30 to 800 Boehringer Units (BLU) were considered to be non-specific (background).

Table 3 Determination of kinetic constants for antibody-EpCAM interactions.

\begin{tabular}{lccc}
\hline Antibody & $\mathbf{k}_{\text {ass }}\left(\mathbf{M}^{-1} \mathbf{s}^{-1}\right)$ & $\mathbf{k}_{\text {diss }}\left(\mathbf{s}^{-1}\right)$ & $\mathbf{K}_{\mathbf{D}} \mathbf{( M )}$ \\
\hline HO-3 & $5.4 \pm 1.6 \times 10^{4}$ & $2.7 \pm 0.8 \times 10^{-5}$ & $5.5 \times 10^{-10} \pm 0.19$ \\
Catumaxomab & $6.1 \pm 1.5 \times 10^{4}$ & $3.3 \pm 0.3 \times 10^{-5}$ & $5.6 \times 10^{-10} \pm 0.12$ \\
\hline
\end{tabular}

Interaction between the ECD of native EpCAM and mAb was measured by surface plasmon resonance. Both the parental $m A b \quad H O-3$ and the trAb variant catumaxomab displayed high-affinity binding with slow off rates.

binding, indicated by reduced-off rates, were observed (data not shown).

\section{Recognition of EpCAM by HO-3 is independent of EpCAM's glycosylation status}

The amino acid sequence of EpCAM comprises three putative N-glycosylation sites (NGT/S) (Chong and Speicher, 2001). Differential usage of these $\mathrm{N}$-glycosylation sequences results in the generation of EpCAM variants of different sizes ranging from 37 to $42 \mathrm{kDa}$. Notably, a hyperglycosylation pattern was observed in head and neck carcinoma when compared with healthy EpCAMpositive epithelium (Pauli et al, 2003).

EpCAM was strongly glycosylated in $77 \%$ of cases of head and neck carcinomas studied $(n=44)$. When comparing pairs of carcinomas and EpCAM-positive thyroid tissue from the same patients $(n=26)$, a significant hyperglycosylation of EpCAM in tumours was observed in $80.7 \%$ of specimen. Hence, we wondered whether protein glycosylation might eventually play a role in antibody recognition, as this would have serious repercussions on therapeutic application.

Point mutations were sequentially inserted into the extracellular domain of wild-type EpCAM to exchange asparagine residues in each $\mathrm{N}$-glycosylation consensus site to an alanine $(N \rightarrow A)$ to generate a triple mutant (N74/111/198A). This EpCAM triple mutant lacking all consensus asparagine residues (N74A, N111A, N198A) was unglycosylated, as shown on glycostaining (Figure 3A). Thereafter, independent bulk cultures of EpCAMwt and N74/111/198A stable HEK293 transfectant were stained with $\mathrm{mAb} \mathrm{HO}-3$ or $\mathrm{C} 215$ and measured in a flow cytometer. EpCAM was detected by both the HO-3 and $\mathrm{C} 215$ antibodies and independently of the glycosylation status, that is EpCAMwt and N74/111/198A were equally well bound (Figure $3 \mathrm{~B}$ ). Hence, binding of $\mathrm{HO}-3$ to EpCAM is not affected by the glycosylation status of the protein.

\section{Catumaxomab, but not HO-3, efficiently mediates tumour cell killing in vitro}

The capacity of HO-3 or the therapeutic trAb catumaxomab to induce the killing of EpCAM-positive tumour cells by immune 
A
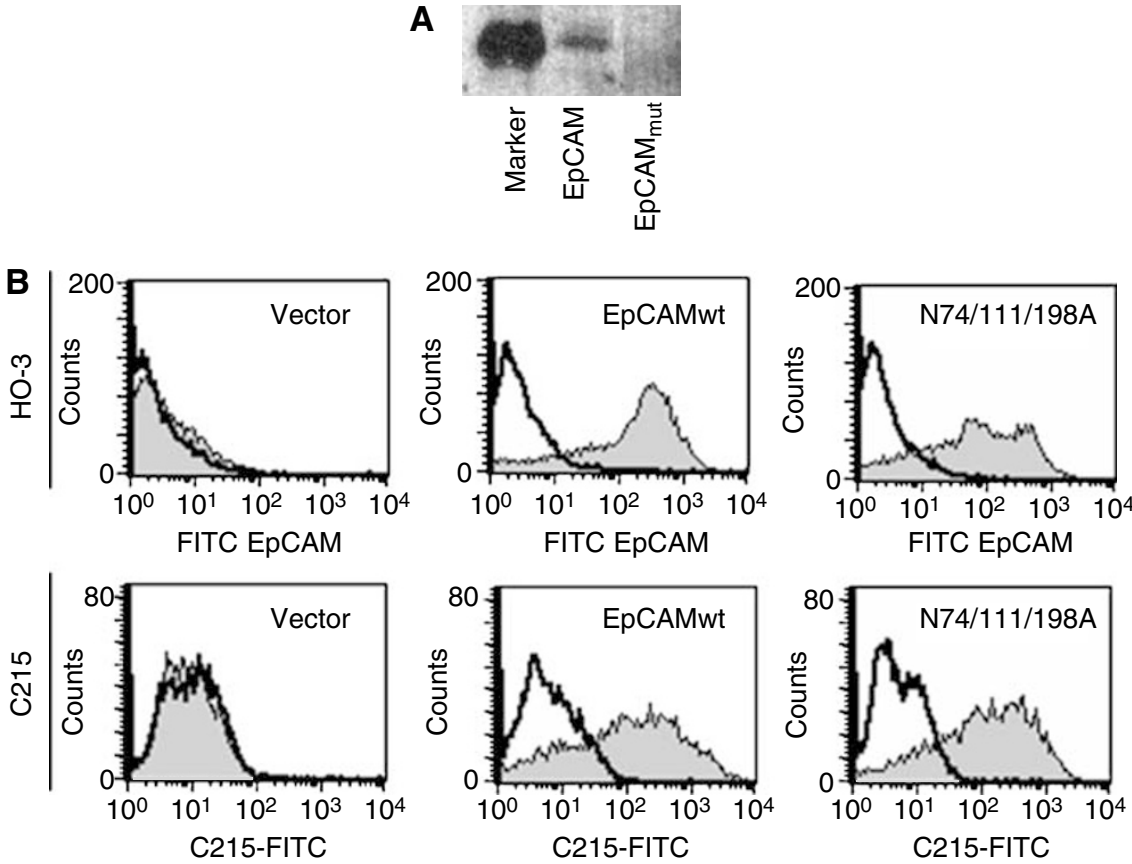

Figure $3 \mathrm{HO}-3$ and C2I5 recognise EpCAM independently of the glycosylation status. (A) HEK293 cells were stably transfected with an expression vector for wild-type EpCAM and a triple mutant lacking all glycosylation sites (EpCAM mut). Equal amounts of cell lysates of each cell lines were separated by I0\% SDS-PAGE and subjected to glycostaining. Staining was assessed with the FLA 5000 scanning device (Fuji). M represents an internal marker provided by the manufacturers. (B) HEK293 cells stably expressing wild-type EpCAM, a triple mutant lacking all N-glycosylation consensus sites (N74/I I I/I98A; EpCAM $M_{\text {mut }}$ ), or the empty vector for a control were stained with $\mathrm{HO}-3$ (grey curves; upper panels) or C2 I 5 (grey curves; lower panels) in combination with a FITC-conjugated secondary antibody. As a control, primary antibody was omitted (black line). All data are representative results from three independent experiments.

effector cells was compared in vitro. The cytotoxic potential of PBMC was assessed by XTT staining after co-cultivation of PBMC and EpCAM-positive tumour targets for 3 days. Significant tumour cell eradication was observed after addition of trAb catumaxomab at low concentration of $0.1 \mathrm{ng} \mathrm{ml}^{-1}$. Tumour cells were quantitatively killed at concentrations greater than $10 \mathrm{ng} \mathrm{ml}^{-1}$ (Figure 4). EpCAM-independent killing induced by CD20-specific trifunctional control antibody Bi20 was approximately $40 \%$. Similar results were obtained using PBMC from different donors $(n=4)$. In sharp contrast, HO-3 mediated a weak killing efficiency only at a favourable effector to target ratio of $50: 1$ and at concentrations as high as $100 \mathrm{ng} \mathrm{ml}^{-1}$. As expected, HO-3-mediated killing was specific since mouse IgG2a isotype control antibody did not display any anti-tumoral activity. In summary, catumaxomab and HO-3 exhibit a strong potency difference with regard to their ability to induce the killing of EpCAM-positive cancer cells in vitro.

\section{DISCUSSION}

Soon after its discovery, the high frequency of EpCAM overexpression in many epithelial tumours suggested it as an attractive target for antibody-based therapies (Herlyn et al, 1979; Gottlinger et al, 1986a; Riethmuller et al, 1994). Since then, there have been a plethora of therapeutic approaches guided by the idea of targeting tumour cells via EpCAM. These strategies include the use of murine and humanised monoclonal antibodies, retroviral constructs, vaccination protocols, as well as bispecific and trifunctional antibodies (Balzar et al, 1999; Armstrong and Eck, 2003; Kufer et al, 2004; Baeuerle and Gires, 2007).

Here, we characterise the new mAb HO-3 that constitutes the EpCAM-binding arm of the corresponding therapeutic trAb catumaxomab. The binding features of this mouse IgG2a antibody

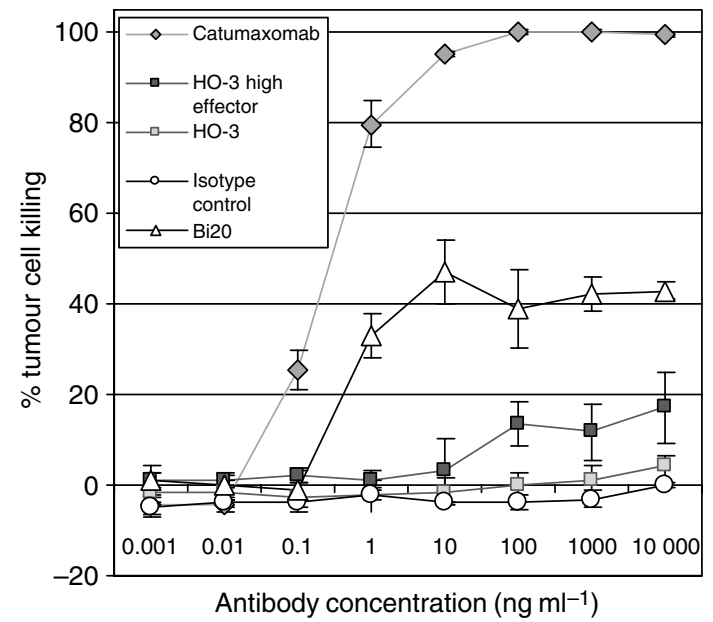

Figure $4 \mathrm{TrAb}$ catumaxomab is 1000-fold more potent than $\mathrm{HO}-3$ in eliciting PBMC-mediated killing of EpCAM-positive carcinoma cells. Comparison of trAb and mAb mediated cytotoxicity against EpCAMpositive tumour cells in vitro. HCT-8 tumour cells and PBMCs were co-cultured at ratios of $10: I$ in the presence of trAbs catumaxomab (antiEpCAM $\times$ anti-CD3), Bi2O (anti-CD2O $\times$ anti-CD3) or mAb HO-3 at the indicated concentrations. Additional approaches with high effector ratios of 50: I were performed for $\mathrm{HO}-3$ and mouse IgG2a isotype control MmT I (anti-mouse Thy-1.2). After 3 days, tumour cell killing was measured by XTT staining. Alloreactivity of PBMC without antibody was not significant (0-2\%). Data points display mean values of four determinations with s.d. Data are representative results from four independent experiments using PBMCs from different donors.

are described in detail. Studies with EpCAM deletion mutants and binding assays in the presence of the competing $\mathrm{mAb} C 215$ pointed toward a recognition site within the EGF-like domain $I$ in the 
extracellular domain of EpCAM. This was confirmed by finemapping the antigenic epitope through the screening of an overlapping peptide library which revealed a discontinuous epitope with two major binding sites within the EGF I and the EGF II domains, respectively. A third minor binding site located C-terminally to EGF II was also identified. Thus, HO-3, just like the great majority of EpCAM-specific antibodies, targets EpCAM mainly within the EGF like domains, which represent the most immunogenic components of this protein (Balzar et al, 1999). Driven by the knowledge of differential glycosylation of EpCAM in normal vs carcinoma tissue (Pauli et al, 2003), we assessed the dependence of HO-3 binding on the glycosylation status of the molecule. A potential impact of post-translational modification of EpCAM on the binding of therapeutic antibodies available so far remained entirely unexplored. Nevertheless, this may be of major importance as suggested by the tumour antigen MUC-1 whose hypoglycosylated variant expressed in tumour tissue is differentially recognised by mucin-specific antibodies (Grinstead et al, 2002; Karsten et al, 2005; Li and Cozzi, 2007). However, HO-3 binding to EpCAM was entirely independent of the presence of glycosyl residues on the target protein. Of note, the glycosylation grade of EpCAM derived from head and neck squamous cell carcinomas varied, and it is as yet unclear whether weakly or nonglycosylated EpCAM variants may appear on other tumours. Taking this into account, mAbs such as HO-3 that are insensitive to the glycosylation status of EpCAM have an expanded potency to target a great variety of EpCAM-positive tumours.

Certainly, the most interesting aspect of new antibodies against EpCAM is their therapeutic value. Upto now, edrecolomab (Panorex ${ }^{\circledR}$ ) is one of the best characterised EpCAM-specific therapeutic antibodies (Gottlinger et al, 1986b; Goodwin et al, 1987). However, the clinical benefit of this mouse IgG2a mAb is limited. Having first shown impressive reduction in mortality in Duke's C colorectal cancer patients compared with the control arm consisting of surgery alone, edrecolomab proved to be less effective than chemotherapy in a proximate phase III study (Riethmuller et al, 1994; Punt et al, 2002). The low affinity of the antibody is one critically discussed aspect. Improved treatment results with the strongly binding $\mathrm{mAb} 323 / \mathrm{A} 3$ in a mouse xenograft tumour model speak in favour of the clinical development of highaffinity anti-EpCAM therapeutic antibodies (Velders et al, 1995). However, the maximum tolerated dose of the high-affinity EpCAM-targeting $\mathrm{mAb}$ ING-1, as determined in a dose escalation study, turned out to be low, with only $0.1 \mathrm{mg} \mathrm{kg}^{-1} i$.v. Additionally, severe toxicity in the form of acute pancreatitis occurred at higher concentrations (de Bono et al, 2004). Keeping the affinity low but improving ADCC effector function by chimaerisation or generation of fully human IgG1 antibodies may be a more promising method. With adecatumumab (MT201), such a novel human IgG1 anti-EpCAM antibody is presently in clinical development (Naundorf et al, 2002). However, common mAb effector functions such as ADCC and CDC may not be strong enough in terms of cancer therapy. High antibody dosages and continued administration are required to compensate for the endogenous serum IgG that competes for binding to $\mathrm{Fc} \gamma$ receptors on immune effector cells, and complement inhibitors that are expressed on tumour cells (Gorter and Meri, 1999; Preithner et al, 2006).

TrAbs represent a novel and promising alternative to improve anti-cancer efficacy. Owing to the simultaneous recruitment and activation of $\mathrm{T}$ cells and accessory immune cells, a variety of effector functions, such as ADCC, phagocytosis, and T-cell mediated cytotoxicity, are induced in a combinatorial fashion (Zeidler et al, 1999, 2000; Riesenberg et al, 2001). This may lead to a 1000-fold increase in anti-tumour potency as was demonstrated in the present study. The trAb catumaxomab (removab ${ }^{\circledR}$ ), which is based on the mAb HO-3 and displays specificities for EpCAM and $\mathrm{CD} 3$, is far more potent in inducing tumour cell killing by PBMC than is HO-3 alone. Besides the trAb-mediated concerted attack by different types of effector cells, the high affinity of the anti-EpCAM binding arm may contribute to its effectiveness as an anti-tumour agent. Not only for HO-3, but also for monovalently binding catumaxomab, we measured a remarkably low dissociation constant of $5.6 \times 10^{-10} \mathrm{M}$. As EpCAM is not tumour-specific, but rather is tumour-associated and also expressed on normal epithelial tissue (Balzar et al, 1999), high affinity conventional mAbs may be disadvantageous, with therapeutic concentrations that cause severe adverse events. This is different with trAbs, which are administered in the clinic at very low dosages in the microgram range. However, to guarantee effective and stable opsonisation of tumour cells, a very high-affinity and a slow-off rate of the therapeutic antibody is required, especially when administered at non-saturating concentrations. Hence, we propose that the highaffinity and the assessed off rate of only $3.3 \pm 0.3 \times 10^{-5} \mathrm{~s}^{-1}$ of the anti-EpCAM targeting arm is one of the crucial features of catumaxomab.

The therapeutic effectiveness of this novel trAb has not only been demonstrated in vitro and in different in vivo tumour models (Lindhofer et al, 1996; Ruf and Lindhofer, 2001; Schmitt et al, 2004). Catumaxomab's clinical benefit was recently verified when it was used to treat patients suffering from malignant ascites (Heiss et al, 2005). In this prospective study, catumaxomab was applied intraperitoneally, was well tolerated, and effectively diminished the local tumour cell burden and ascites fluid accumulation.

Biodistribution studies in EpCAM transgenic mice suggested preferential access of EpCAM-specific mAbs to tumour cells in spite of a background expression of EpCAM on healthy tissue (McLaughlin et al, 2001). This demonstrates the suitability of using EpCAM for antibody-based cancer therapy, in principle. In conclusion, the application of high-affinity and effective trAbs administered locally at very low concentrations may re-open the therapeutic window for immunotherapy of EpCAM expressing tumours.

\section{ACKNOWLEDGEMENTS}

We thank Susanne Wosch, Melanie Goelden, and Sandra Huber for expert technical assistance.

\section{REFERENCES}

Armstrong A, Eck SL (2003) EpCAM: a new therapeutic target for an old cancer antigen. Cancer Biol Ther 2: 320-326

Baeuerle PA, Gires O (2007) EpCAM (CD326) finding its role in cancer. Br J Cancer 96: 417-423

Balzar M, Winter MJ, de Boer CJ, Litvinov SV (1999) The biology of the 17-1A antigen (Ep-CAM). J Mol Med 77: 699-712

Bjork P, Jonsson U, Svedberg H, Larsson K, Lind P, Dillner J, Hedlund G, Dohlsten M, Kalland T (1993) - Isolation, partial characterization, and molecular cloning of a human colon adenocarcinoma cell-surface glycoprotein recognized by the C215 mouse monoclonal antibody. J Biol Chem 268: $24232-24241$

Chong JM, Speicher DW (2001) Determination of disulfide bond assignments and N-glycosylation sites of the human gastrointestinal carcinoma antigen GA733-2 (CO17-1A, EGP, KS1-4, KSA, and Ep-CAM). J Biol Chem 276: $5804-5813$

de Bono JS, Tolcher AW, Forero A, Vanhove GF, Takimoto C, Bauer RJ, Hammond LA, Patnaik A, White ML, Shen S, Khazaeli MB, Rowinsky EK, LoBuglio AF (2004) ING-1, a monoclonal antibody 
targeting Ep-CAM in patients with advanced adenocarcinomas. Clin Cancer Res 10: $7555-7565$

Gastl G, Spizzo G, Obrist P, Dunser M, Mikuz G (2000) Ep-CAM overexpression in breast cancer as a predictor of survival. Lancet 356: $1981-1982$

Goodwin RA, Tuttle SE, Bucci DM, Jewell SD, Martin EW, Steplewski Z (1987) Tumor-associated antigen expression of primary and metastatic colon carcinomas detected by monoclonal antibody 17-1A. Am J Clin Pathol 88: $462-467$

Gorter A, Meri S (1999) Immune evasion of tumor cells using membranebound complement regulatory proteins. Immunol Today 20: 576-582

Gottlinger HG, Funke I, Johnson JP, Gokel JM, Riethmuller G (1986b) The epithelial cell surface antigen 17-1A, a target for antibody-mediated tumor therapy: its biochemical nature, tissue distribution and recognition by different monoclonal antibodies. Int J Cancer 38: 47-53

Gottlinger HG, Funke I, Johnson JP, Gokel JM, Riethmuller G (1986a) The epithelial cell surface antigen 17-1A, a target for antibody-mediated tumor therapy: its biochemical nature, tissue distribution and recognition by different monoclonal antibodies. Int J Cancer 38: 47-53

Grinstead JS, Koganty RR, Krantz MJ, Longenecker BM, Campbell AP (2002) Effect of glycosylation on MUC1 humoral immune recognition: NMR studies of MUC1 glycopeptide-antibody interactions. Biochemistry 41: $9946-9961$

Gutzmer R, Li W, Sutterwala S, Lemos MP, Elizalde JI, Urtishak SL, Behrens EM, Rivers PM, Schlienger K, Laufer TM, Eck SL, Marks MS (2004) A tumor-associated glycoprotein that blocks MHC class II-dependent antigen presentation by dendritic cells. J Immunol 173: $1023-1032$

Heiss MM, Strohlein MA, Jager M, Kimmig R, Burges A, Schoberth A, Jauch KW, Schildberg FW, Lindhofer H (2005) Immunotherapy of malignant ascites with trifunctional antibodies. Int J Cancer 117: 435-443

Herlyn D, Herlyn M, Steplewski Z, Koprowski H (1979) Monoclonal antibodies in cell-mediated cytotoxicity against human melanoma and colorectal carcinoma. Eur J Immunol 9: 657-659

Karsten U, von Mensdorff-Pouilly S, Goletz S (2005) What makes MUC1 a tumor antigen? Tumour Biol 26: 217-220

Kiewe P, Hasmuller S, Kahlert S, Heinrigs M, Rack B, Marme A, Korfel A, Jager M, Lindhofer H, Sommer H, Thiel E, Untch M (2006) Phase I trial of the trifunctional anti-HER $2 \times$ anti-CD3 antibody ertumaxomab in metastatic breast cancer. Clin Cancer Res 12: 3085-3091

Kremmer E, Thierfelder S, Kummer U, Lederer R, Mysliwietz J (1989) Neutralization of immunosuppression by antibodies against variable as well as constant regions of monoclonal anti-Thy-1 xenoantibodies and their ability to be suppressed by initial $\mathrm{T}$ cell depletion. Transplantation 47: $641-646$

Kufer P, Lutterbuse R, Baeuerle PA (2004) A revival of bispecific antibodies. Trends Biotechnol 22: 238-244

Li Y, Cozzi PJ (2007) MUC1 is a promising therapeutic target for prostate cancer therapy. Curr Cancer Drug Targets 7: 259-271

Lindhofer H, Menzel H, Gunther W, Hultner L, Thierfelder S (1996) Bispecific antibodies target operationally tumor-specific antigens in two leukemia relapse models. Blood 88: 4651-4658

Lindhofer H, Mocikat R, Steipe B, Thierfelder S (1995) Preferential speciesrestricted heavy/light chain pairing in rat/mouse quadromas. Implications for a single-step purification of bispecific antibodies. J Immunol 155: $219-225$

Litvinov SV, Velders MP, Bakker HA, Fleuren GJ, Warnaar SO (1994) EpCAM: a human epithelial antigen is a homophilic cell-cell adhesion molecule. J Cell Biol 125: 437-446

McLaughlin PM, Harmsen MC, Dokter WH, Kroesen BJ, van der MH, Brinker MG, Hollema H, Ruiters MH, Buys CH, de Leij LF (2001) The epithelial glycoprotein 2 (EGP-2) promoter-driven epithelial-specific expression of EGP-2 in transgenic mice: a new model to study carcinoma-directed immunotherapy. Cancer Res 61: 4105-4111

Munz M, Kieu C, Mack B, Schmitt B, Zeidler R, Gires O (2004) The carcinoma-associated antigen EpCAM upregulates c-myc and induces cell proliferation. Oncogene 23: $5748-5758$

Naundorf S, Preithner S, Mayer P, Lippold S, Wolf A, Hanakam F, Fichtner I, Kufer P, Raum T, Riethmuller G, Baeuerle PA, Dreier T (2002) In vitro and in vivo activity of MT201, a fully human monoclonal antibody for pancarcinoma treatment. Int J Cancer 100: 101-110
Osta WA, Chen Y, Mikhitarian K, Mitas M, Salem M, Hannun YA, Cole DJ, Gillanders WE (2004) EpCAM is overexpressed in breast cancer and is a potential target for breast cancer gene therapy. Cancer Res 64: 5818-5824

Pauli C, Munz M, Kieu C, Mack B, Breinl P, Wollenberg B, Lang S, Zeidler R, Gires O (2003) Tumor-specific glycosylation of the carcinomaassociated epithelial cell adhesion molecule EpCAM in head and neck carcinomas. Cancer Lett 193: 25-32

Preithner S, Elm S, Lippold S, Locher M, Wolf A, da Silva AJ, Baeuerle PA, Prang NS (2006) High concentrations of therapeutic IgG1 antibodies are needed to compensate for inhibition of antibody-dependent cellular cytotoxicity by excess endogenous immunoglobulin G. Mol Immunol 43: $1183-1193$

Punt CJ, Nagy A, Douillard JY, Figer A, Skovsgaard T, Monson J, Barone C, Fountzilas G, Riess H, Moylan E, Jones D, Dethling J, Colman J, Coward L, MacGregor S (2002) Edrecolomab alone or in combination with fluorouracil and folinic acid in the adjuvant treatment of stage III colon cancer: a randomised study. Lancet 360: 671-677

Reineke U, Kramer A, Schneider-Mergener J (1999) Antigen sequence- and library-based mapping of linear and discontinuous protein-proteininteraction sites by spot synthesis. Curr Top Microbiol Immunol 243: $23-36$

Riesenberg R, Buchner A, Pohla H, Lindhofer H (2001) Lysis of prostate carcinoma cells by trifunctional bispecific antibodies (alpha EpCAM $\times$ alpha CD3). J Histochem Cytochem 49: 911-917

Riethmuller G, Schneider-Gadicke E, Schlimok G, Schmiegel W, Raab R, Hoffken K, Gruber R, Pichlmaier H, Hirche H, Pichlmayr R (1994) Randomised trial of monoclonal antibody for adjuvant therapy of resected Dukes' C colorectal carcinoma. German Cancer Aid 17-1A Study Group [see comments]. Lancet 343: 1177-1183

Ruf P, Lindhofer H (2001) Induction of a long-lasting antitumor immunity by a trifunctional bispecific antibody. Blood 98: 2526-2534

Schmitt M, Schmitt A, Reinhardt P, Thess B, Manfras B, Lindhofer H, Riechelmann H, Wiesneth M, Gronau S (2004) Opsonization with a trifunctional bispecific (alphaCD3 $\times$ alphaEpCAM) antibody results in efficient lysis in vitro and in vivo of EpCAM positive tumor cells by cytotoxic T lymphocytes. Int J Oncol 25: 841-848

Spizzo G, Obrist P, Ensinger C, Theurl I, Dunser M, Ramoni A, Gunsilius E, Eibl G, Mikuz G, Gastl G (2002) Prognostic significance of Ep-CAM AND Her-2/neu overexpression in invasive breast cancer. Int J Cancer 98: $883-888$

Spizzo G, Went P, Dirnhofer S, Obrist P, Moch H, Baeuerle PA, MuellerHolzner E, Marth C, Gastl G, Zeimet AG (2006) Overexpression of epithelial cell adhesion molecule (Ep-CAM) is an independent prognostic marker for reduced survival of patients with epithelial ovarian cancer. Gynecol Oncol 103: 483-488

Stemmler HJ, Salat C, Lindhofer H, Menzel H, Untch M, Kahlert S, Konecny G, Sauer H, Ledderose G, Heinemann V, Kolb HJ (2005) Combined treatment of metastatic breast cancer (MBC) by high-dose chemotherapy (HDCT) and bispecific antibodies: a pilot study. Anticancer Res 25: $3047-3054$

Varga M, Obrist P, Schneeberger S, Muhlmann G, Felgel-Farnholz C, Fong D, Zitt M, Brunhuber T, Schafer G, Gastl G, Spizzo G (2004) Overexpression of epithelial cell adhesion molecule antigen in gallbladder carcinoma is an independent marker for poor survival. Clin Cancer Res 10: $3131-3136$

Velders MP, van Rhijn CM, Briaire IH, Fleuren GJ, Warnaar SO, Litvinov SV (1995) Immunotherapy with low and high affinity monoclonal antibodies $17-1 \mathrm{~A}$ and $323 / \mathrm{A} 3$ in a nude mouse xenograft carcinoma model. Cancer Res 55: $4398-4403$

Went P, Vasei M, Bubendorf L, Terracciano L, Tornillo L, Riede U, Kononen J, Simon R, Sauter G, Baeuerle PA (2006) Frequent high-level expression of the immunotherapeutic target Ep-CAM in colon, stomach, prostate and lung cancers. Br J Cancer 94: 128-135

Zeidler R, Mysliwietz J, Csanady M, Walz A, Ziegler I, Schmitt B, Wollenberg B, Lindhofer H (2000) The Fc-region of a new class of intact bispecific antibody mediates activation of accessory cells and NK cells and induces direct phagocytosis of tumour cells. Br J Cancer 83: 261-266 Zeidler R, Reisbach G, Wollenberg B, Lang S, Chaubal S, Schmitt B, Lindhofer H (1999) Simultaneous activation of T cells and accessory cells by a new class of intact bispecific antibody results in efficient tumor cell killing. J Immunol 163: 1246-1252 